Revue

Revue de l'histoire des religions

de Ihistoire des religions

3 | 2016

Varia

Heliand, L'évangile de la mer du Nord, préface de Michel ROUCHE, texte présenté et traduit par Eric VANNEUFVILLE

Turnhout, Brepols (« Miroir du Moyen Âge »), 2009

Jens Schneider

\title{
OpenEdition
}

Journals

Édition électronique

URL : http://journals.openedition.org/rhr/8606

DOI : 10.4000/rhr.8606

ISSN : 2105-2573

Éditeur

Armand Colin

Édition imprimée

Date de publication : 1 septembre 2016

Pagination : 443-446

ISBN : 978-2-200-93061-5

ISSN : 0035-1423

Référence électronique

Jens Schneider, « Heliand, L'évangile de la mer du Nord, préface de Michel Rouche, texte présenté et traduit par Eric VANNEUfVILLE », Revue de l'histoire des religions [En ligne], 3 | 2016, mis en ligne le 06 octobre 2016, consulté le 25 septembre 2020. URL : http://journals.openedition.org/rhr/8606 ; DOI : https://doi.org/10.4000/rhr.8606

Ce document a été généré automatiquement le 25 septembre 2020.

Tous droits réservés 


\section{Heliand, L'évangile de la mer du Nord, préface de Michel ROUCHE, texte présenté et traduit par Eric VANNEUFVILLE}

Turnhout, Brepols (« Miroir du Moyen Âge »), 2009

Jens Schneider

\section{RÉFÉRENCE}

Heliand, L'évangile de la mer du Nord, préface de Michel Rouche, texte présenté et traduit par Eric VANNEUfVILLE, Turnhout, Brepols (« Miroir du Moyen Âge »), 2009, 488 p., $21 \mathrm{~cm}$, $50 €$, ISBN 978-2-503-52866-3.

Ce livre semble issu d'une entreprise de valorisation d'un texte numérisé dans le cadre du projet «Wulfila» à l'université d'Anvers. On a réimprimé une paraphrase des évangiles en vieux-saxon dans l'édition proposée par Eduard Sievers en 1878, fondée sur le manuscrit de Munich, avec une traduction française qui parait être la première du poème dans son intégralité, conservé en fragment, et comprenant 5983 vers dans ce manuscrit. Entreprise ambitieuse à résultat douteux : Éric Vanneufville est sans doute un historien accompli dans le domaine des Flandres mais ses compétences ne s'étendent visiblement pas à la littérature et aux langues germaniques $d u \mathrm{IX}^{\mathrm{e}}$ siècle, sans compter que les coquilles abondent et que de manière générale, le volume est peu soigné. L'« aperçu bibliographique » (p. 485-487) fournit des références incomplètes et privilégie les études et les éditions de la première moitié $d u x^{e}$ siècle; parmi les quelques sources indiquées (9 titres) et les ouvrages de synthèse et catalogues d'exposition, on cherchera en vain l'édition de référence (Heliand und Genesis, éd. O. Behaghel, $10^{e}$ éd. par B. Taeger, Tübingen, 1996) ou bien une publication scientifique consacrée à l'Heliand (dernier état de la recherche par W. Haubrichs dans 
Althochdeutsche und altsächsische Literatur, sous la dir. de R. Bergmann, Berlin-New York, 2013, p. 154-163).

L'introduction n'est pas dépourvue d'aperçus anachroniques qu'il est aisé de critiquer mais qui étonnent tout de même. La langue bas-allemande du Haut Moyen Âge n'est pas le plattdeutsch d'aujourd'hui mais le niederdeutsch, nuance importante car elle ne comprend pas seulement le vieux saxon mais aussi le bas francique, le frison et, dans la logique d'une linguistique diachronique, l'anglo-saxon ou vieil anglais; s'interroger sur la «nationalité de l'auteur» de l'Heliand (p. 8) donne une mauvaise impression des représentations identitaires au Haut Moyen Âge, de même l'emploi du terme " gentilhomme germain » dont on se demande ce qu'il signifie au juste (p. 12).

Le lecteur de l'introduction aurait apprécié quelques remarques au sujet des manuscrits conservés de ce texte. Pas un mot sur la découverte du fragment de Leipzig qui a fait l'objet de plusieurs articles, certes parus peu de temps avant la présente traduction. Précisons donc qu'il faut supposer l'existence d'un manuscrit copié au milieu du IX siècle dont faisaient partie les fragments $\mathrm{P}$ (anciennement à Prague, aujourd'hui à Berlin, DHM, R 56/2537) et L (Leipzig, UB, ms. Thomas 4073). Les informations assemblées par le traducteur à propos de la langue du texte et de son auteur supposé restent superficielles et semblent surtout avoir profité du Manuel de l'Allemand du Moyen Âge, des origines au XIVe siècle, publié par Alfred Jolivet et Fernand Mossé en 1942. Cette question a pourtant été l'objet d'études importantes, à commencer par une étude de Thomas Klein publiée en 1977. Tenant compte de ces travaux, l'hypothèse d'un arrièrefond frison avancée par E. Vanneufville semble difficilement soutenable. Le lecteur découvrira aussi diverses observations, dont la plus importante semble être la reprise de l'analyse linguistique de J. J. van Weringh, mal référencée (il s'agit probablement d'une contribution au cahier Liudger, Bernlef, Heliand ..., sous la dir. de K. Sierksma, Muiderberg, 1984), pour qui quelques fragments du texte de l'Heliand, nommés d'après le lieu de leur découverte en 1977 à Straubing en Bavière, seraient « tout à fait proches du "Vieux-Frison" » (p. 21). Cette mise en contexte frisonne de l'œuvre vieux-saxonne qu'est le Heliand se fait sur une base très mince, pratiquement dépourvue de textes qui permettraient la comparaison linguistique. Elle s'inscrit dans les réflexions d'E. Vanneufville à propos d'une langue commune partagée par les locuteurs le long de la mer du Nord qui aurait donné lieu à une « aire anglo-frisonne » (p. 20), réflexions qui rappellent fatalement les hypothèses d'un espace culturel celtique, voire d'un royaume des deux côtés de la Manche, émises par quelques auteurs bretons du $\mathrm{xx}^{\mathrm{e}}$ siècle. E. Vanneufville fait bien de rapprocher le Heliand des quelques textes conservés en vieil-anglais de la même période mais il ne renvoie pas aux éditions de ces textes et cite comme seule référence un autre manuel de 1927. Il oublie notamment de mentionner la "Génèse vieil-anglaise ", texte en lien étroit avec la poésie vieux-saxonne, qui a d'ailleurs également fait l'objet d'un projet de numérisation (The Junius Manuscript, éd. G. Ph. Krapp, New York-Londres, 1931; A Digital Facsimile of Oxford, Bodleian Library, MS. Junius 11, éd. B. J. Muir et N. Kennedy, Oxford, Bodleian digital texts 12004 [CD-ROM]).

4 En ce qui concerne la terminologie, le lecteur n'est pas sûr que les mots « anglo-saxon » et "vieil-anglais", qui ne sont que deux termes pour désigner la même chose, soient utilisés ici de manière vraiment synonyme. En outre, peu d'informations sont données sur la structure du poème organisé en 71 parties (Fitten) et sur les traits stylistiques de ce texte qui représente le témoin le plus important de l'allitération germanique (Stabreim) en langue vieil-allemande. La technique recherchée employée par l'auteur 
inconnu qui consiste à lier deux vers par des «crochets " (Hakenreim) semble quand même démontrer qu'il s'agit d'un poète expérimenté en la matière. L'auteur du commentaire nous propose, en note de lecture, des observations intéressantes qui mettent en relation la description des répressions romaines en Israël dans le Heliand avec celles imposées par les Francs en pays saxon, y compris l'installation de représentants du pouvoir central. Plus précisément, E. Vanneufville rapproche le massacre des innocents attribué à Hérode aux grandes pertes qu'ont dû subir les Saxons pendant les trente ans de guerre de conquête franque. Ajoutons les importantes mesures de déplacement de grandes parties de la population saxonne, qualifiées de déportation par l'historien Matthias Springer (M. Springer, Die Sachsen, Stuttgart, 2004). Dans ce contexte, on s'étonne également de l'« attitude désormais apaisante à l'égard des nouveaux convertis» (p.14) qu'aurait pris Charlemagne. En revanche, il est intéressant de suivre l'auteur dans ses observations sur le cadre naturel, le climat, et l'ambiance de la vie quotidienne qui sont remplacés dans le Heliand par les conditions de vie d'une Europe marquée par la mer du Nord (p. 15-16).

5 Prenons enfin quelques passages pour vérifier la traduction. Le début souffre du maintien du texte proposé par l'édition de Sievers, contre les éditions parues depuis 1878. La situation textuelle n'est pas très claire pour ces premières lignes, ce qui ne peut toutefois excuser la faute classique du traducteur qui est d'adopter des «faux amis » : on peut difficilement traduire $\bmod (v .1)$ par " courage » même si le terme de l'allemand actuel Mut semble s'y prêter; en revanche gibodscip (v. 8) et gibod (v. 14) sont traduits par "précepte ", en dépit de l'entrée du lexique proposé en annexe où l'on trouvera gebod, traduit convenablement par «ordre» (p. 463). La présentation des quatre évangélistes qui, selon le texte vieux-saxon, furent les seuls à être choisis pour rédiger l'évangile dans un livre, n'est pas très réussie : "Ils étaient ainsi choisis, ceux qui allaient mettre par écrit en un livre l'évangile et aussi les nombreux préceptes de Dieu, la sainte parole céleste » (p. 33). Notons que « les nombreux préceptes de Dieu » ne rend pas très bien so manag gibod godes (v. 14). Autre exemple, le récit du Sermon sur la montagne. Pourquoi avoir choisi « Maître " pour traduire le participe uualdand, celui qui exerce le pouvoir (v. 1281), de même «le Christ Maître» pour uualdand Crist (v. 1325) ? La traduction ne distingue pas iro rikia (v. 1308), « leur royaume» (celui des fidèles, plus précisément le royaume de «ceux qui se lamentaient de leurs mauvaises actions ", p. 121), du royaume du Seigneur (rikia drohtines, v. 1309, ou sinum rikea, « son royaume ", v. 1316). Salige sind oc undar thesaro managon thiodu / thie hebbiad iro herta gihrenod (v. 1314-1315) est traduit comme suit: «Bienheureuses aussi les nombreuses personnes qui ont purifié leur cœur» (p. 121). Rappelons ici l'existence du verbe sind «sont » et précisons surtout que sont bienheureux « parmi ce peuple nombreux / ceux qui... ». L'historien linguiste, comme l'historien tout court, sera sensible à la mention de thioda (peuple) au deuxième quart du Ix ${ }^{\mathrm{e}}$ siècle.

6 Arrêtons-nous pour évoquer la citation donnée en amont de la traduction (p. 31). E. Vanneufville met en exergue huit lignes de texte en français actuel qu'il présente dans une note de bas de page comme « emprunté aux huit premières lignes relatées par l'éminent historien belge Lodewyck De Baecker ». Ce n'est pas l'absence d'une référence qui est regrettable, mais plutôt le fait que l'auteur n'a pas conscience de citer deux passages séparés (ici unifiés) d'un texte qui est parmi les plus connus de la période vieux-haut-allemande, à savoir la dédicace d'une autre célèbre paraphrase des évangiles par Otfrid de Wissembourg au roi Louis le Pieux (Otfrid von Weißenburg, 
Evangelienbuch, éd. W. Kleiber et E. Hellgardt, t. I.1 : Edition nach dem Wiener Codex 2687, Tübingen, 2004, fol. $1^{\mathrm{r}}-3^{\mathrm{r}}$, v. 1-2 et 89-90).

7 Pour finir, la traduction est peu précise et on y rencontre souvent de vraies erreurs. Le texte original en vieux-saxon ne correspond pas aux critères d'une édition critique. L'introduction n'est pas une présentation scientifique du document primordial que représente le Heliand; elle ne renseigne pas sur la situation des cinq manuscrits conservés en partie ou en totalité. Les informations concernant le contexte historique, les hypothèses avancées par rapport au public et l'auteur de ce texte mettent en évidence qu'E. Vanneufville ne tient pas compte de la bibliographie importante qui existe à propos de ce texte poétique. Le livre a le mérite de rendre accessible une traduction française du Heliand et il a l'honnêteté de la présenter avec le texte original. Quant à la valorisation du travail de numérisation du texte médiéval achevé à l'université d'Anvers, on ne peut que recommander d'adopter «une résignation germanique face au destin » (p. 14).

\section{AUTEURS}

\section{JENS SCHNEIDER}

Université Paris-Est Marne-la-Vallée. 